\title{
Translational and Clinical Applications of Dental Stem Cell-Derived Exosomes
}

Zizhao Mai ${ }^{1+}$, Huan Chen ${ }^{1 \dagger}$, Yu Ye ${ }^{2,3}$, Ziyu Hu ${ }^{4}$, Wenjuan Sun ${ }^{5}$, Li Cui $^{1,6 *}$ and Xinyuan Zhao ${ }^{1 *}$

${ }^{1}$ Stomatological Hospital, Southern Medical University, Guangzhou, China, ${ }^{2}$ Institute of Stomatology, Nanjing Medical University, Nanjing, China, ${ }^{3}$ Key Laboratory of Oral Diseases of Jiangsu Province, Stomatological Institute, Nanjing Medical University, Nanjing, China, ${ }^{4}$ Department of Pediatrics, Nanjing Jinling Stomatology Hospital, Nanjing, China, ${ }^{5}$ Department of Stomatology, The Third Affiliated Hospital, Sun Yat-sen University, Guangzhou, China, ${ }^{6}$ UCLA School of Dentistry, Los Angeles, CA, United States

\section{OPEN ACCESS}

Edited by: Anne George,

University of Illinois at Chicago,

United States

Reviewed by:

Amar M. Singh,

University of Georgia, United States

Philippe Bourin,

Independent researcher, Toulouse,

France

*Correspondence:

Xinyuan Zhao

zhaoxinyuan1989@smu.edu.cn

Li Cui

licui@smu.edu.cn

${ }^{\dagger}$ These authors have contributed equally to this work

Specialty section:

This article was submitted to

Stem Cell Research,

a section of the journal

Frontiers in Genetics

Received: 31 July 2021 Accepted: 07 October 2021 Published: 26 October 2021

Citation:

Mai Z, Chen H, Ye Y, Hu Z, Sun W, Cui $L$ and Zhao $X$ (2021) Translational and Clinical Applications of Dental

Stem Cell-Derived Exosomes.

Front. Genet. 12:750990.

doi: 10.3389/fgene.2021.750990
Mesenchymal stem cells (MSCs) are promising seed cells in tissue repair and regeneration due to their featured properties of self-renewal and multipotency. However, a growing body of evidence has demonstrated that MSCs exert biological functions mainly through secreting exosomes. Exosomes, which contain RNA, proteins, lipids, and metabolites, are new players in regulating many fundamental processes and play important roles in regenerative medicine. Exosomes not only mimic the effects of their parent cells but also possess many advantages such as high drug loading capacity, low immunogenicity, excellent biocompatibility, and low side effects. Currently, a total of 6 different dental stem cells (DSCs) including dental pulp stem cells (DPSCs), stem cells from exfoliated deciduous teeth (SHEDs), periodontal ligament stem cells (PDLSCs), dental follicle progenitor cells (DFPCs), stem cells from apical papilla (SCAPs) and gingival mesenchymal stem cells (GMSCs) have been isolated and identified. DSC-derived exosomes (DSC-Exos) are actively involved in intercellular communication, anti-inflammation, osteogenesis, angiogenesis, immunomodulation, nurturing neurons, and promoting tumor cell apoptosis. In this review, we will critically review the emerging role and clinical application potential of DSC-Exos.

Keywords: dental stem cells, exosomes, regenerative medicine, tissue repair, clinical application

\section{INTRODUCTION}

Mesenchymal stem cells (MSCs) are multipotent progenitor cells that can be isolated from different tissues including but not limited to, bone marrow (Friedenstein et al., 1966), adipose tissue (Gruber et al., 2010), placenta (In 't Anker et al., 2004), umbilical cord (Secco et al., 2008), hair follicle (Bajpai, Mistriotis, and Andreadis 2012), palatine tonsil (Ryu et al., 2012), amniotic fluid (In 't Anker et al., 2003), fetal blood and liver (Campagnoli et al., 2001). Accumulative evidence has demonstrated that MSCs are capable of self-renewal, multipotent differentiation (Minguell et al., 2001; Munmun and Witt-Enderby 2021), regulating immune and inflammatory responses (Uccelli et al., 2008), and suppressing apoptosis and oxidative stress (Tsubokawa et al., 2010). More importantly, numerous pre-clinical studies have shown that MSCs hold promise in treating a wide range of diseases, including cancer, liver disease, cartilage repair, heart failure, stroke, neurological disorders, diabetes mellitus, autoimmune diseases, Duchenne muscular dystrophy, ocular surface diseases (Lodi et al., 2011; Lu et al., 2021). Over the past 10 years, more than 1000 MSC-based clinical trials have been 

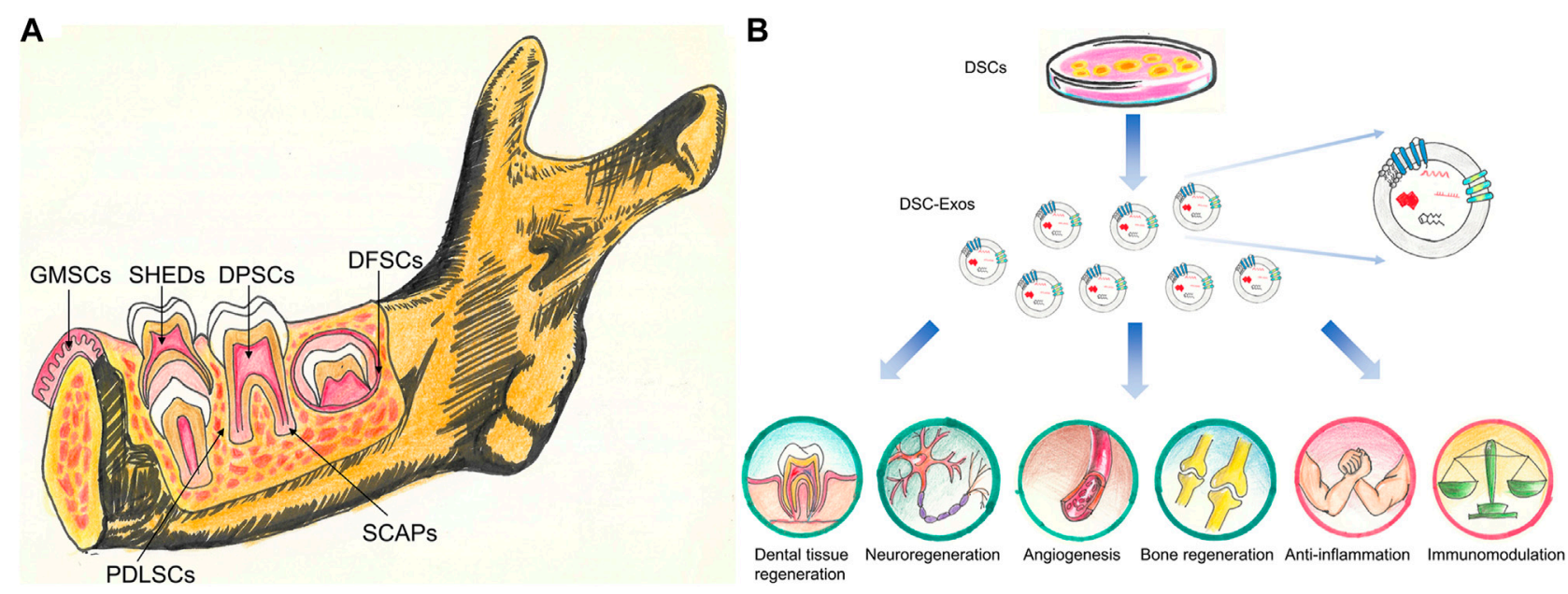

FIGURE 1 | (A) Stem cell populations derived from different dental tissues. DPSCs, stem cells from dental pulp; SHEDs, stem cells from human exfoliated deciduous teeth; PDLSCs, stem cells from PDL; DFSCs, stem cells from dental follicle; SCAPs, stem cells from the apical papilla; GMSCs, stem cells from gingival tissues. (B) The potential clinical application of DSC-Exos. DSC-Exos hold great promise for dental tissue regeneration, neuroregeneration, angiogenesis, bone regeneration, anti-inflammation and immunomodulation.

conducted with recruitment of approximately 50,000 patients due to the safety of autogenous stem cells (Pittenger et al., 2019). Interestingly, accumulative evidence has suggested that the therapeutic effects of transplanted MSCs largely depend on the secretome of MSCs rather than the MSCs themselves (Phinney and Pittenger 2017).

Distinct from other types of cell therapy, MSC-based therapy achieves the therapeutic effects not only through direct cell-cell contacts but also by releasing secretome-derived bioactive factors (Levy et al., 2020). Recently, the MSC secreted extracellular vesicles (MSC-EVs), which include exosomes, microvesicles, and apoptotic bodies, have been suggested as a viable cell-free therapeutic alternative for MSCs (Jarrige et al., 2021). Compared to the cellular therapies, the MSC-EVs-based therapy offer many advantages such as high drug loading capacity, high specificity, low immunogenicity, excellent biocompatibility, high stability, lack of cytotoxicity, competitive price, and efficient intercellular communication. Therefore, MSC-EVs-based therapy, especially using exosomes, has emerged as a promising therapeutic tool for tissue repair and regeneration. In this review, we critically focus on the potential value of exosomes derived from dental stem cells (DSC-Exos) for treating oral and systemic diseases.

\section{FEATURED PROPERTIES OF DENTAL STEM CELLS AND EXOSOMES \\ Dental Stem Cells (DSCs)}

Currently, a total of six different dental stem cells (DSCs) including dental pulp stem cells (DPSCs), stem cells from exfoliated deciduous teeth (SHEDs), periodontal ligament stem cells (PDLSCs), dental follicle progenitor cells (DFPCs), stem cells from apical papilla (SCAPs) and gingival mesenchymal stem cells
(GMSCs) have been isolated and identified (Zhang et al., 2009; Sedgley and Botero 2012) (Figure 1A).

DPSCs, the first characterized DSCs, were isolated from the human dental pulp in 2000 (Gronthos et al., 2000). The MSC-like properties of DPSCs enable them to differentiate into multiple cell lineages such as neural-like cells, osteoblasts, adipocytes, and chondrocytes, and form mineralized tissue, blood vessels, and nerve tissues in vivo (Gronthos et al., 2000; Gronthos et al., 2002; Victor and Reiter 2017). Besides, DPSCs have also been demonstrated to regenerate dentin and functional dental pulp with vasculature and nerves (Iohara et al., 2014).

SHEDs, obtained from human exfoliated deciduous teeth by Miura et al. (2003), is a population of highly proliferative cells capable of differentiating into odontoblasts and endothelial cells (Miura et al., 2003). After being injected into human root canals, the transplanted SHEDs could differentiate into functional odontoblasts and form dentin-like tissues (Rosa et al., 2013). Interestingly, SHEDs remained viable following transplantation into the mouse brain and expressed neural markers (Miura et al., 2003).

Similarly, PDLSCs are named according to their tissue of origin, and isolated from the periodontal ligament which is a soft connective tissue between the teeth and alveolar bone. PDLSCs are promising seed cells for the restoration of periodontal tissues and are capable of differentiating into adipocytes, chondrocytes, osteoblasts, cardiac myocytes and neural cells (Gay et al., 2007; Tomokiyo et al., 2019). PDLSCs interact tightly with the periodontitis niche in a positive feedback loop. The injured PDLSCs may aggravate the disrupted periodontal tissue homeostasis, while bacterial infections and subsequent host immune responses affect the functional properties of resident PDLSCs by shaping the periodontal microenvironment (Zhang Y. et al., 2021). 
TABLE 1 | The common and different properties for each type of DSCs.

\begin{tabular}{|c|c|c|c|}
\hline $\begin{array}{l}\text { Cell } \\
\text { type }\end{array}$ & Advantage & Weakness & Similarity \\
\hline DPSCs & $\begin{array}{l}\text { Formation of dentin-pulp-like complex, } \\
\text { Source for reparative dentin }\end{array}$ & $\begin{array}{l}\text { Essential to be extracted from healthy adult teeth, } \\
\text { differential growth rates, cell morphologies, and sizes }\end{array}$ & Fibroblast-like morphology \\
\hline SHEDS & $\begin{array}{l}\text { Formation of dentin-like tissue or pulp-like } \\
\text { tissue, The most proliferative DSCs }\end{array}$ & Unable to form dentin-pulp-like complex & $\begin{array}{l}\text { Common markers: } \mathrm{CD}^{+}{ }^{+}, \mathrm{CD}^{+} 9^{+}, \mathrm{CD}^{+} 3^{+}, \mathrm{CD}^{+} \text {, } \\
\text { CD105+, CD106+, CD146+, Stro-1+, CD34 }{ }^{-}, \mathrm{CD} 45^{-} \text {, } \\
\text { CD11b-, CD14 }{ }^{-} \text {CD19 }{ }^{-}, \mathrm{CD} 79 a-, \text { and HLA-DR-High } \\
\text { clonogenic potential }\end{array}$ \\
\hline PDLSCs & $\begin{array}{l}\text { Formation of PDL-cementum-like } \\
\text { construction }\end{array}$ & Lack odontogenic potential & Multilineage differentiation capacity \\
\hline DFPCs & $\begin{array}{l}\text { Formation of alveolar bone, Formation of } \\
\text { PDL-cementum-like construction }\end{array}$ & Lack odontogenic potential & \\
\hline SCAPS & $\begin{array}{l}\text { Maintenance of root maturation, } \\
\text { Formation of dentin-pulp-like complex }\end{array}$ & Not easily obtainable & \\
\hline GMSCs & Easy to isolate, Long-term stability & Lack odontogenic potential & \\
\hline
\end{tabular}

DFPCs were first isolated from the dental follicle surrounding the impacted third molar tooth germ and identified by Morsczeck et al. (2005). DFPCs not only have better immunomodulatory and anti-apoptotic effects on the immune system than DPSCs and SHED, but also exhibit greater osteogenic properties than SHED and DPSCs as the osteogenic-related markers such as Runx2 and DSPP are highly expressed in DFPCs (Zhu et al., 2018). In addition, DFPCs were able to differentiate into cementoblasts in vivo (Handa et al., 2002). Therefore, DFSCs are a promising alternative source for dental hard tissue regeneration.

SCAPs are isolated from the apical papilla of immature permanent teeth, and play a critical role in tooth root development and dentin regeneration (Sonoyama et al., 2008). The high telomerase activity of SCAPs makes them a better choice of dentin regeneration compared to DPSCs (Sonoyama et al., 2006). In addition, SCAPs are able to form cementum/PDL-like complex in vivo (Han et al., 2010). Moreover, SCAPs have been shown to be low immunogenicity and possess immunomodulatory functions, which make them an attractive and promising therapeutic tool for tissue regeneration (Ding et al., 2010).

GMSCs were first isolated from healthy gingival tissues and characterized by Zhang et al., in 2009 (Zhang et al., 2009). GMSCs can differentiate into adipocytes, chondrocytes, and endothelial cells, and have shown great promise for nerve regeneration. Besides, GMSCs exerted anti-proliferative and pro-apoptotic effects on oral cancer cells both in vitro and in vivo (Ji et al., 2016).

To the best of our knowledge, it is still extremely difficult to distinguish various sources of DSCs on a molecular level. All different types of DSCs are derived from migrating neural crest cells, which are originated from the embryonic ectoderm germ layer (Pisciotta et al., 2020). The fibroblast-like DSCs share similar surface marker expression profiles, and have high clonogenic potential and multipotent differentiation capacity. A recent study suggested that calreticulin might be a promising biomarker for distinguishing DPSCs and GMSCs. We have summarized the similarity, advantages, and weaknesses of each type of DSCs in Table 1.

\section{Exosomes}

Exosomes, with a size range of 40-160 nm (average $100 \mathrm{~nm}$ ), are originated from the endosomal system by inward budding of the endosomal membrane (Xu F. et al., 2020; Yoon et al., 2020). Ultracentrifugation, size-based isolation techniques, immunoaffinity capture-based techniques, exosome precipitation, and microfluidic-based isolation techniques are utilized to isolate exosomes (Doyle and Wang 2019). They are found in abundance in body fluids including breast milk, saliva, blood, and urine (Vlassov et al., 2012). This type of extracellular vesicles was initially thought of as waste products (Wolf 1967). However, accumulative evidence has demonstrated that exosomes carry many bioactive molecules including nucleic acids, proteins, lipids, metabolites (Zhang Y. et al., 2020; Kalluri and LeBleu 2020). These encapsulated materials are transported to neighboring or distant cells selectively, which contribute to cell-cell communication, signal transduction, immune response modulation, antigen presentation, and epigenetic reprogramming of recipient cells (Janockova et al., 2021). The biological functions of exosomes are heavily dependent on physiological/pathological conditions of originating tissues or cells at the time of exosome secretion, and the surface receptors of the recipient cells (AlcayagaMiranda et al., 2016). As shown in Figure 1B, the DSCderived exosomes might represent an ideal therapeutic tool for tissue repair and regeneration as well as treating other systemic diseases.

\section{TRANSLATIONAL AND CLINICAL APPLICATIONS OF DENTAL STEM CELL-DERIVED EXOSOMES \\ DPSC-Exos}

The high osteo/odonto-induction capability and easy availability of DPSCs-derived exosomes (DPSC-Exos) make them highly attractive in regenerative medicine (Imanishi et al., 2021). In addition, aged DPSCs still have active cellular metabolism and secrete functional exosomes which 
can penetrate the blood-brain barrier, indicating DPSC-Exos might be an effective drug carrier for the treatment of various diseases, especially for neurological disorders like Parkinson's disease (PD) (Haney et al., 2015; Iezzi et al., 2019). For instance, DPSC-Exos were deemed as a suitable carrier to deliver tumor suppressor miR-34a to inhibit the proliferation of breast cancer cells (Vakhshiteh et al., 2021). In terms of odontogenic differentiation, Huang et al. revealed that DPSC-Exos attached to biomaterials by binding to matrix proteins like fibronectin and type I collagen (Huang et al., 2016). Besides, DPSC-Exos, especially those isolated from DPSCs cultured under an odontogenic differentiation environment, increased the expression of genes indispensable for odontogenic differentiation of naïve DPSCs in vitro and promoted the regeneration of dental pulp-like tissues in vivo (Huang et al., 2016; Swanson et al., 2020). Similarly, compared to exosomes isolated from DPSCs cultured undergrowth state, DPSC-Exos obtained under odontogenic conditions exhibited better performance for trigging odontogenic differentiation of DPSCs by activating the TGF- $\beta 1 /$ smads signaling pathway (Huang et al., 2016; Hu et al., 2019). Exosomes derived from both mineralizing DPSCs and an immortalized murine odontoblast cell line (MDPC-23) were superior to traditional glass-ionomer cement for forming the reparative dentin bridge (Swanson et al., 2020). It seems that lipopolysaccharide (LPS) treatment can significantly alter the biological functions of DPSCs-Exos. Li et al. demonstrated that LPS-preconditioned DPSC derived exosomes (LPSDPSC-Exos) promoted the proliferation, migration, and odontogenic differentiation of Schwann cells ( $\mathrm{Li}$ et al., 2021). Similarly, LPS-DPSC-Exos were shown to promote angiogenesis by facilitating the proliferation, migration, and tube formation abilities of human umbilical vein endothelial cells (HUVECs) in vitro through changing the microRNA (miRNA) expression profile and increasing the levels of kinase-insert domain-containing receptor and vascular endothelial growth factor (Huang et al., 2021). DPSC-Exos also exhibits strong anti-inflammatory and immunomodulatory effects. For instance, DPSC-Exos facilitated alveolar bone reconstruction and periodontal epithelium healing in a mouse model of periodontitis via delivering exosomal miR-1246 (Shen et al., 2020). DPSCExos exerted immunomodulatory effects by suppressing the differentiation of CD4+T cells into T helper 17 cells (Th17) and facilitated the transformation of $\mathrm{CD} 4+\mathrm{T}$ cells into regulatory $\mathrm{T}$ cells (Tregs), leading to the increased level of anti-inflammatory cytokines (Ji et al., 2019). In addition to oral diseases, DPSC-Exos based therapy is promising for treating systemic diseases. For example, exosomes derived from DPSC might be more suitable in the treatment of neurodegenerative diseases than MSCs from mesodermal tissues such as bone marrow or adipose tissues (Wang et al., 2019). Exosomes secreted from miR-140-5p overexpressing DPSCs promoted the expression of genes related to chondrogenic differentiation and exerted anti-apoptotic effects both in vitro and in vivo, which represented a potentially novel therapeutic strategy for osteoarthritis (Lin et al., 2021).

\section{SHED-Exos}

The applications of SHED-derived exosomes (SHED-Exos) can be classified into the following three categories: promotion of osteogenesis, neurotrophic property and anti-inflammatory function. In terms of osteogenesis, SHED-Exos promoted osteogenic differentiation of PDLSCs by upregulating key genes and signaling pathways related to osteogenesis (Wang A. et al., 2020). Similarly, SHED-Exos was shown to have the potency for mobilizing naïve BMMSCs, resulting in enhancing bone regeneration (Luo et al., 2021). Wei et al. revealed that SHED-Exo promoted osteogenesis and suppressed adipogenesis of bone marrow mesenchymal stem cells (BMMSCs) by decreasing lipid droplets and the expression of the adipogenic marker PPAR $\gamma$ (Wei et al., 2020). SHED-Exos promoted neovascularization of HUVECs and osteogenic differentiation of BMMSCs, and this regulatory effect could be counteracted by adding AMPK inhibitor, indicating that the AMPK signaling pathway might involve in mediating the pro-angiogenic effects and pro-bone regeneration activities of SHED-Exos (Wu et al., 2019). Concerning neurotrophic property, exosomes isolated from SHEDs grown on the laminin-coated three-dimensional alginate micro-carriers protected dopaminergic neurons from 6hydroxy-dopamine induced apoptosis, whereas exosomes from SHEDs grown under standard culture conditions had no such effects (Jarmalaviciute et al., 2015). Li et al. injected SHED-Exos into the traumatic brain injury (TBI) rat model and observed that SHED-Exos contributed to rat motor functional restoration and cortical lesion reduction by shifting microglia polarization ( $\mathrm{Li}$ et al., 2017). Narbute et al. showed that SHED-Exos significantly improved the gait impairments and contralateral rotations in the unilateral 6-hydroxydopamine (6-OHDA) rat model of PD (Narbute et al., 2019). Collectively, SHED-Exos are promising therapeutic tools for neurological disorders like PD. In terms of anti-inflammatory effect, SHED-Exos significantly suppressed the carrageenan-induced acute inflammation in vivo (Pivoraite et al., 2015). Similarly, Luo et al. showed that SHED-Exos markedly reduced the inflammation in chondrocytes derived from the temporomandibular joint through delivering miR100-5p (Luo et al., 2019).

\section{PDLSC-Exos}

The major regulatory functions of PDLSCs-derived exosomes (PDLSC-Exos) include angiogenesis, anti-inflammation, and osteogenesis. Inflammation led to increasing exosome secretion in PDLSCs, and exosomes derived from inflamed PDLSC promoted angiogenesis of HUVECs by upregulating the expression of vascular specific marker CD31 and VEGFA (Zhang Z. et al., 2020). The exosomes isolated from PDLSCs that were exposed to the LPS-induced periodontitis environment demonstrated good anti-inflammatory ability by modulating the balance of $\mathrm{T}$ helper cell 17 (Th17)/regulatory $\mathrm{T}$ cell (Treg) through the miR-155-5p/SIRT1 pathway (Zheng et al., 2019). In terms of osteogenesis, PDLSC-Exos possess the capacity for inducing osteogenic differentiation of BMMSCs via regulating AMPK signaling, MAPK signaling, and insulin signaling pathways (Liu et al., 2020). P2X7R overexpressing PDLSCsderived conditional medium and exosomes markedly 
TABLE 2 | The potential clinical application of DSC-Exos.

\begin{tabular}{|c|c|c|c|c|c|}
\hline Origin & Administration & Recipients & Application & Refs & $\begin{array}{l}\text { The potentially } \\
\text { most suitable } \\
\text { application of } \\
\text { specific DSC-Exos }\end{array}$ \\
\hline DPSCs & In vitro & Breast cancer cells & Cancer & Vakhshiteh et al. (2021) & Pulp and dentin regeneration \\
\hline- & In vitro & $\mathrm{CD}^{+} \mathrm{T}$ cells & Immunomodulation & Ji et al. (2019) & \\
\hline- & In vitro & Schwann cells & Pulp regeneration & Li et al. (2021) & \\
\hline- & In vitro & HUVECs & Angiogenesis & Huang et al. (2021) & \\
\hline- & In vivo & Athymic nude mice & Pulp regeneration & Huang et al. (2016) & \\
\hline- & In vivo & Rat pulpotomy model & Dentin regeneration & Swanson et al. (2020) & \\
\hline- & In vivo & Periodontitis model & Periodontitis & Shen et al. (2020) & \\
\hline- & In vivo & Osteoarthritis model & Osteoarthritis & Lin et al. (2021) & \\
\hline SHEDs & In vitro & PDLSCs & Osteogenic differentiation & Wang et al. (2020b) & Parkinson's disease \\
\hline- & In vitro & BMMSCs & Migration promotion & Luo et al. (2021) & Neuroregeneration \\
\hline- & In vitro & Neurons & Parkinson's disease & Jarmalaviciute et al. (2015) & \\
\hline- & In vitro & Chondrocytes & Osteoarthritis & Luo et al. (2019) & \\
\hline- & In vivo & Periodontal defect & Bone regeneration & Wu et al. (2019) & \\
\hline- & In vivo & BMMSCS & Bone regeneration & Wei et al. (2020) & \\
\hline- & In vivo & Traumatic brain injury model & Traumatic brain injury & Li et al. (2017) & \\
\hline- & In vivo & Parkinson's disease model & Parkinson's disease & Narbute et al. (2019) & \\
\hline- & In vivo & Mouse paw edema & Anti-inflammation & Pivoraite et al. (2015) & \\
\hline PDLSCs & In vitro & $\mathrm{CD}^{+} \mathrm{T}$ cells & Immunomodulation & Zheng et al. (2019) & Periodontitis induced bone loss \\
\hline- & In vitro & BMMSCs & Osteogenic differentiation & Liu et al. (2020) & \\
\hline- & In vitro & PDLSCs & Osteogenic differentiation & Xu et al. (2020b) & \\
\hline- & In vitro & HUVECs & Angiogenesis & Zhang et al. (2020a) & \\
\hline SCAPs & In vivo & Immunodeficient mice & Dentinogenesis & Zhuang et al. (2020) & Not enough evidences for evaluation \\
\hline- & In vivo & Critical-size defects & Soft tissue regeneration & Liu et al. (2021) & \\
\hline GMSCs & In vitro & Pre-osteoblast & Osteogenic differentiation & Jiang and Xu (2020) & Taste bud regeneration and would healing \\
\hline- & In vitro & Macrophages & Anti-inflammation & Zhang et al. (2021b) & \\
\hline- & In vivo & Schwann and DRG cells & Nerve repair & Rao et al. (2019) & \\
\hline- & In vivo & Skin defect model & Would healing & Shi et al. (2017) & \\
\hline- & In vivo & Periodontitis model & Bone regeneration & Nakao et al. (2021) & \\
\hline- & In vivo & Tongue defect model & Taste bud regeneration & Zhang et al. (2019) & \\
\hline DFPCs & NA & $\mathrm{NA}$ & Not evaluated yet & $\mathrm{NA}$ & NA \\
\hline
\end{tabular}

improved the osteogenic capacity of PDLSCs in the inflammatory microenvironment by delivering miR-3679-5p, miR-6515-5p, and miR-6747-5p (Xu X. Y. et al., 2020). Interestingly, the circRNA and IncRNA expression profile was significantly altered in PDLSCs-exos during the osteogenic differentiation of PDLSCs, indicating that the exosomal non-coding RNAs might play a critical role in regulating PDLSCs osteogenesis (Xie et al., 2021). Collectively, PDLSC-Exos is beneficial to the maintenance of periodontal homeostasis by promoting proliferation, angiogenesis, and osteogenesis as well as regulating the inflammatory responses.

\section{SCAP-Exos}

SCAP-derived exosomes (SCAP-Exos) also show great potential for oral tissue regeneration. Zhuang et al. demonstrated that SCAP-Exos promoted the dentinogenesis of BMMSCs both in vitro and in vivo, indicating that SCAP-Exos might represent a potential therapeutic tool for dentine-pulp complex regeneration (Zhuang et al., 2020). Through injecting into the palatal gingival complex critical-size defects (CSD) of mice, SCAP-Exos significantly improved angiogenesis and soft tissue regeneration (Liu et al., 2021). In terms of mechanism, SCAPExos promoted filopodium formation, migration, and cytoskeletal reorganization of endothelial cells via delivering exosomal Cdc42. Wang et al. compared the piRNA expression profiles between SCAP-Exos and BMMSC-Exos (Wang M. et al., 2020). The differentially expressed piRNAs were found to be closely associated with many important biological functions such as catalytic activity, metabolic processes, cellular processes, binding, and biological regulation, suggesting that piRNAs might play a crucial role in regulating the molecular activities of exosomes.

\section{GMSC-Exos}

Although currently few studies are available regarding the potential therapeutic applications of GMSC-Exos, they hold a great promise for tissue regeneration. The pre-osteoblasts MC3T3-E1 treated with GMSC-Exos were found deep Alizarin red staining, increased ALP activity, and upregulated expression of osteogenic genes, suggesting that GMSC-Exos facilitated the osteogenic differentiation of MC3T3-E1 (Jiang and Xu 2020). The exosomes isolated from tumor necrosis factor-alpha (TNF- $\alpha$ ) preconditioned GMSC-Exos induced the polarization of antiinflammatory M2 macrophage by improving the secretion of GMSC-Exos and increased the exosomal expression of CD73 (Nakao et al., 2021). In a high-lipid microenvironment, GMSCExos suppressed lipid accumulation, transformed proinflammatory macrophages into anti-inflammatory phenotype, 
and decrease the secretion and expression of inflammatory factors including IL-6, IL-1 $\beta$, TNF- $\alpha$, and cluster of differentiation 86 (Zhang Z. et al., 2021). Besides, GMSC-Exos presented anti-osteoclastogenic activity and suppressed inflammatory bone loss by delivering miR-1260b (Nakao et al., 2021). Rao et al. combined GMSC-Exos with biodegradable chitin conduits and injected the composite into the rat sciatic nerve defect model. The results showed that GMSC-Exos enhanced the proliferation of Schwann cells and the growth of the dorsal root ganglion neuron axon as well as promoting the formation of nerve fibers and myelin, which subsequently contributed to restoring motor skills, nerve conduction function, and muscle movement (Rao et al., 2019). GMSC-Exos also promoted healing of diabetic skin defects by facilitating re-epithelialization, collagen remodeling, angiogenesis, and nerve growth in a diabetic rat skin defect model (Shi et al., 2017). Combining GMSC-Exos with small intestinal submucosa-extracellular matrix promoted taste bud regeneration and tongue lingual papillae recovery in a rat tongue defect model (Zhang et al., 2019). We have summarized the currently available evidence regarding the potential clinical application of DSC-Exos (Table 2).

\section{FUTURE PERSPECTIVES AND CHALLENGES}

DSC-Exos hold great promise in tissue repair and regeneration as well as treating other diseases. However, to the best of our knowledge, there is still not enough evidence for evaluating and comparing the molecular differences, biological functions, and therapeutic applications among different types of DSC-Exos as well as between DSC-Exos and other MSCs-Exos. Compared to exosomes derived from other MSCs, DSC-Exos might possess their own advantages. For instance, DPSC-Exos have stronger immunomodulatory, anti-necrotic, and anti-apoptotic effects than BMMSC-Exos (Venugopal et al., 2018; Ji et al., 2019). The currently available evidence demonstrates that DSC-Exos might share similar limitations and weaknesses. Compared to the exosomes from BMMSCs and adipose tissue-derived MSCs, the isolation of a sufficient amount of DSCs-Exos is still an obstacle for hindering their therapeutic applications (Stanko et al., 2018). More importantly, compared to autologous BMMSC from bone marrow aspirate, DSCs are mainly collected from non-renewable exfoliated deciduous teeth, third molars, and teeth extracted by orthodontic treatment and irreversible periodontitis, or discarded

\section{REFERENCES}

Alcayaga-Miranda, F., Varas-Godoy, M., and Khoury, M. (2016). Harnessing the Angiogenic Potential of Stem Cell-Derived Exosomes for Vascular Regeneration. Stem Cell Int. 2016, 1-11. doi:10.1155/2016/3409169

Bajpai, V. K., Mistriotis, P., and Andreadis, S. T. (2012). Clonal Multipotency and Effect of Long-Term In Vitro Expansion on Differentiation Potential of Human Hair Follicle Derived Mesenchymal Stem Cells. Stem Cel Res. 8 (1), 74-84. doi:10.1016/j.scr.2011.07.003

Campagnoli, C., Roberts, I. A. G., Kumar, S., Bennett, P. R., Bellantuono, I., and Fisk, N. M. (2001). Identification of Mesenchymal Stem/progenitor Cells in tissues after dental surgery. Therefore, it is challenging to obtain DSC-Exos in time when they are needed. Only under the condition that the unnecessary tissues like exfoliated deciduous teeth and third molar were stored previously by a stable and longterm approach, the applications of DSC-Exos might be popularized on a large scale. In addition, there are a lack of standardized and accepted approaches for storage, transport and large-scale production of DSCs-Exos, which has significantly affected their clinical applications. Mover, determining the most appropriate dose of exosomes under different pathological conditions, avoiding the off-target effects, and ensuring sufficient biological safety of DSC-Exos need to be urgently addressed in the coming pre-clinical studies and/or clinical trials.

\section{CONCLUSIONS}

DSCs mainly exert their therapeutic effects by the secretion of exosomes via a paracrine mechanism. Compared to DSCs, DSCExos possess unique advantages such as high drug loading capacity, high specificity, low immunogenicity, excellent biocompatibility, easily obtainable, low side effects, and nanoscale size. In addition, DSC-Exos have been shown to regulate many important biological processes including intercellular communication, anti-inflammation, osteogenesis, angiogenesis, immunomodulation, nurturing neurons, and promoting tumor cell apoptosis. Although there are still many barriers to translation into clinical practice, DSC-Exos is emerging as a promising and practical therapeutic approach for tissue repair and regeneration.

\section{AUTHOR CONTRIBUTIONS}

Conceptualization and funding acquisition, $\mathrm{XZ}$ and LC; writingoriginal draft and editing, ZM, HC, YY, ZH, WS, XZ, and LC; All authors have read and agreed to the submitted version of the manuscript.

\section{FUNDING}

This work was supported by the National Natural Science Foundation of China (Nos 81901006).
Human First-Trimester Fetal Blood, Liver, and Bone Marrow. Blood 98 (8), 2396-2402. doi:10.1182/blood.v98.8.2396

Carnevale, G., Pisciotta, A., Bertoni, L., Vallarola, A., Bertani, G., and Mecugni, D. (2020). Neural Crest Derived Stem Cells from Dental Pulp and ToothAssociated Stem Cells for Peripheral Nerve Regeneration. Neural Regen. Res. 15 (3), 373-381. doi:10.4103/1673-5374.266043

Ding, G., Liu, Y., An, Y., Zhang, C., Shi, S., Wang, W., et al. (2010). Suppression of T Cell Proliferation by Root Apical Papilla Stem Cells In Vitro. Cells Tissues Organs 191 (5), 357-364. doi:10.1159/000276589

Doyle, L., and Wang, M. (2019). Overview of Extracellular Vesicles, Their Origin, Composition, Purpose, and Methods for Exosome Isolation and Analysis. Cells 8 (7), 727. doi: $10.3390 /$ cells 8070727 
Friedenstein, A. J., Piatetzky-Shapiro, I. I., II, and Petrakova, K. V. (1966). Osteogenesis in Transplants of Bone Marrow Cells. J. Embryol. Exp. Morphol. 16 (3), 381-390. doi:10.1242/dev.16.3.381

Gay, I., Chen, S., and MacDougall, M. (2007). Isolation and Characterization of Multipotent Human Periodontal Ligament Stem Cells. Orthod. Craniofac. Res. 10 (3), 149-160. doi:10.1111/j.1601-6343.2007.00399.x

Gronthos, S., Brahim, J., Li, W., Fisher, L. W., Cherman, N., Boyde, A., et al. (2002). Stem Cell Properties of Human Dental Pulp Stem Cells. J. Dent Res. 81 (8), 531-535. doi:10.1177/154405910208100806

Gronthos, S., Mankani, M., Brahim, J., Robey, P. G., and Shi, S. (2000). Postnatal Human Dental Pulp Stem Cells (DPSCs) In Vitro and Invivo. Proc. Natl. Acad. Sci. 97 (25), 13625-13630. doi:10.1073/pnas.240309797

Gruber, H. E., Deepe, R., Hoelscher, G. L., Ingram, J. A., Norton, H. J., Scannell, B., et al. (2010). Human Adipose-Derived Mesenchymal Stem Cells: Direction to a Phenotype Sharing Similarities with the Disc, Gene Expression Profiling, and Coculture with Human Annulus Cells. Tissue Eng. A 16 (9), 2843-2860. doi:10.1089/ten.TEA.2009.0709

Han, C., Yang, Z., Zhou, W., Jin, F., Song, Y., Wang, Y., et al. (2010). Periapical Follicle Stem Cell: a Promising Candidate for Cementum/periodontal Ligament Regeneration and Bio-Root Engineering. Stem Cell Develop. 19 (9), 1405-1415. doi:10.1089/scd.2009.0277

Handa, K., Saito, M., Tsunoda, A., Yamauchi, M., Hattori, S., Sato, S., et al. (2002). Progenitor Cells from Dental Follicle Are Able to Form Cementum Matrix In Vivo. Connect. Tissue Res. 43 (2-3), 406-408. doi:10.1080/03008200290001023

Haney, M. J., Klyachko, N. L., Zhao, Y., Gupta, R., Plotnikova, E. G., He, Z., et al. (2015). Exosomes as Drug Delivery Vehicles for Parkinson's Disease Therapy. J. Controlled Release 207, 18-30. doi:10.1016/j.jconrel.2015.03.033

Hu, X., Zhong, Y., Kong, Y., Chen, Y., Feng, J., and Zheng, J. (2019). Lineagespecific Exosomes Promote the Odontogenic Differentiation of Human Dental Pulp Stem Cells (DPSCs) through TGF $\beta 1 /$ smads Signaling Pathway via Transfer of microRNAs. Stem Cel Res Ther 10 (1), 170. doi:10.1186/s13287019-1278-x

Huang, C.-C., Narayanan, R., Alapati, S., and Ravindran, S. (2016). Exosomes as Biomimetic Tools for Stem Cell Differentiation: Applications in Dental Pulp Tissue Regeneration. Biomaterials 111, 103-115. doi:10.1016/ j.biomaterials.2016.09.029

Huang, X., Qiu, W., Pan, Y., Li, J., Chen, Z., Zhang, K., et al. (2021). Exosomes from LPS-Stimulated hDPSCs Activated the Angiogenic Potential of HUVECs In Vitro. Stem Cell Int. 2021, 1-15. doi:10.1155/2021/6685307

Iezzi, I., Pagella, P., Pagella, P., Mattioli-Belmonte, M., and Mitsiadis, T. (2019). The Effects of Ageing on Dental Pulp Stem Cells, the Tooth Longevity Elixir. eCM 37, 175-185. doi:10.22203/eCM.v037a11

Imanishi, Y., Hata, M., Matsukawa, R., Aoyagi, A., Omi, M., Mizutani, M., et al. (2021). Efficacy of Extracellular Vesicles from Dental Pulp Stem Cells for Bone Regeneration in Rat Calvarial Bone Defects. Inflamm. Regener 41 (1), 12. doi:10.1186/s41232-021-00163-w

In 't Anker, P. S., Scherjon, S. A., Kleijburg-van der Keur, C., de Groot-Swings, G. M. J. S., Claas, F. H. J., Fibbe, W. E., et al. (2004). Isolation of Mesenchymal Stem Cells of Fetal or Maternal Origin from Human Placenta. Stem Cells 22 (7), 1338-1345. doi:10.1634/stemcells.2004-0058

In 't Anker, P. S., Scherjon, S. A., Kleijburg-van der Keur, C., Noort, W. A., Claas, F. H. J., Willemze, R., et al. (2003). Amniotic Fluid as a Novel Source of Mesenchymal Stem Cells for Therapeutic Transplantation. Blood 102 (4), 1548-1549. doi:10.1182/blood-2003-04-1291

Iohara, K., Murakami, M., Nakata, K., and Nakashima, M. (2014). Age-dependent Decline in Dental Pulp Regeneration after Pulpectomy in Dogs. Exp. Gerontol. 52, 39-45. doi:10.1016/j.exger.2014.01.020

Janockova, J., Slovinska, L., Harvanova, D., Spakova, T., and Rosocha, J. (2021). New Therapeutic Approaches of Mesenchymal Stem Cells-Derived Exosomes. J. Biomed. Sci. 28 (1), 39. doi:10.1186/s12929-021-00736-4

Jarmalavičiūtè, A., Tunaitis, V., Pivoraitè, U., Venalis, A., and Pivoriūnas, A. (2015). Exosomes from Dental Pulp Stem Cells rescue Human Dopaminergic Neurons from 6-Hydroxy-Dopamine-Induced Apoptosis. Cytotherapy 17 (7), 932-939. doi:10.1016/j.jcyt.2014.07.013

Jarrige, M., Frank, E., Herardot, E., Martineau, S., Darle, A., Benabides, M., et al. (2021). The Future of Regenerative Medicine: Cell Therapy Using Pluripotent Stem Cells and Acellular Therapies Based on Extracellular Vesicles. Cells 10 (2), 240. doi:10.3390/cells10020240
Ji, L., Bao, L., Gu, Z., Zhou, Q., Liang, Y., Zheng, Y., et al. (2019). Comparison of Immunomodulatory Properties of Exosomes Derived from Bone Marrow Mesenchymal Stem Cells and Dental Pulp Stem Cells. Immunol. Res. 67 (45), 432-442. doi:10.1007/s12026-019-09088-6

Ji, X., Zhang, Z., Han, Y., Song, J., Xu, X., Jin, J., et al. (2016). Mesenchymal Stem Cells Derived from normal Gingival Tissue Inhibit the Proliferation of Oral Cancer Cells In Vitro and In Vivo. Int. J. Oncol. 49 (5), 2011-2022. doi:10.3892/ ijo.2016.3715

Jiang, S., and Xu, L. (2020). Exosomes from Gingival Mesenchymal Stem Cells Enhance Migration and Osteogenic Differentiation of Pre-osteoblasts. Pharmazie 75 (11), 576-580. doi:10.1691/ph.2020.0652

Kalluri, R., and LeBleu, V. S. (2020). The Biology, Function, and Biomedical Applications of Exosomes. Science 367 (6478), eaau6977. doi:10.1126/ science.aau6977

Levy, O., Kuai, R., Siren, E. M. J., Bhere, D., Milton, Y., Nissar, N., et al. (2020). Shattering Barriers toward Clinically Meaningful MSC Therapies. Sci. Adv. 6 (30), eaba6884. doi:10.1126/sciadv.aba6884

Li, J., Ju, Y., Liu, S., Fu, Y., and Zhao, S. (2021). Exosomes Derived from Lipopolysaccharide-Preconditioned Human Dental Pulp Stem Cells Regulate Schwann Cell Migration and Differentiation. Connect. Tissue Res. 62 (3), 277-286. doi:10.1080/03008207.2019.1694010

Li, Y., Yang, Y.-Y., Ren, J.-L., Xu, F., Chen, F.-M., and Li, A. (2017). Exosomes Secreted by Stem Cells from Human Exfoliated Deciduous Teeth Contribute to Functional Recovery after Traumatic Brain Injury by Shifting Microglia M1/M2 Polarization in Rats. Stem Cel Res Ther 8 (1), 198. doi:10.1186/s13287-0170648-5

Lin, T., Wu, N., Wang, L., Zhang, R., Pan, R., and Chen, Y.-F. (2021). Inhibition of Chondrocyte Apoptosis in a Rat Model of Osteoarthritis by Exosomes Derived from miR-140-5p-overexpressing H-uman D-ental P-ulp S-tem C-ells. Int. J. Mol. Med. 47 (3). doi:10.3892/ijmm.2020.4840

Liu, T., Hu, W., Zou, X., Xu, J., He, S., Chang, L., et al. (2020). Human Periodontal Ligament Stem Cell-Derived Exosomes Promote Bone Regeneration by Altering MicroRNA Profiles. Stem Cell Int. 2020, 1-13. doi:10.1155/2020/ 8852307

Liu, Y., Zhuang, X., Yu, S., Yang, N., Zeng, J., Liu, X., et al. (2021). Exosomes Derived from Stem Cells from Apical Papilla Promote Craniofacial Soft Tissue Regeneration by Enhancing Cdc42-Mediated Vascularization. Stem Cel Res Ther 12 (1), 76. doi:10.1186/s13287-021-02151-w

Lodi, D., Iannitti, T., and Palmieri, B. (2011). Stem Cells in Clinical Practice: Applications and Warnings. J. Exp. Clin. Cancer Res. 30, 9. doi:10.1186/17569966-30-9

Lu, K. H., Lu, P. W. A., Lu, E. W. H., Tang, C. H., Su, S. C., Lin, C. W., et al. (2021). The Potential Remedy of Melatonin on Osteoarthritis. J. Pineal Res. 71, e12762. doi:10.1111/jpi.12762

Luo, L., Avery, S. J., and Waddington, R. J. (2021). Exploring a Chemotactic Role for EVs from Progenitor Cell Populations of Human Exfoliated Deciduous Teeth for Promoting Migration of Naïve BMSCs in Bone Repair Process. Stem Cell Int. 2021, 1-7. doi:10.1155/2021/6681771

Luo, P., Jiang, C., Ji, P., Wang, M., and Xu, J. (2019). Exosomes of Stem Cells from Human Exfoliated Deciduous Teeth as an Anti-inflammatory Agent in Temporomandibular Joint Chondrocytes via miR-100-5p/mTOR. Stem Cel Res Ther 10 (1), 216. doi:10.1186/s13287-019-1341-7

Minguell, J. J., Erices, A., and Conget, P. (2001). Mesenchymal Stem Cells. Exp. Biol. Med. (Maywood) 226 (6), 507-520. doi:10.1177/ 153537020122600603

Miura, M., Gronthos, S., Zhao, M., Lu, B., Fisher, L. W., Robey, P. G., et al. (2003). SHED: Stem Cells from Human Exfoliated Deciduous Teeth. Proc. Natl. Acad. Sci. 100 (10), 5807-5812. doi:10.1073/pnas.0937635100

Morsczeck, C., Götz, W., Schierholz, J., Zeilhofer, F., Kühn, U., Möhl, C., et al. (2005). Isolation of Precursor Cells (PCs) from Human Dental Follicle of Wisdom Teeth. Matrix Biol. 24 (2), 155-165. doi:10.1016/j.matbio.2004.12.004

Munmun, F., and Witt-Enderby, P. A. (2021). Melatonin Effects on Bone: Implications for Use as a Therapy for Managing Bone Loss. J. Pineal Res. 71 (1), e12749. doi:10.1111/jpi.12749

Nakao, Y., Fukuda, T., Zhang, Q., Sanui, T., Shinjo, T., Kou, X., et al. (2021). Exosomes from TNF- $\alpha$-Treated Human Gingiva-Derived MSCs Enhance M2 Macrophage Polarization and Inhibit Periodontal Bone Loss. Acta Biomater. 122, 306-324. doi:10.1016/j.actbio.2020.12.046 
Narbute, K., Pilipenko, V., Pupure, J., Dzirkale, Z., Jonavičè, U., Tunaitis, V., et al. (2019). Intranasal Administration of Extracellular Vesicles Derived from Human Teeth Stem Cells Improves Motor Symptoms and Normalizes Tyrosine Hydroxylase Expression in the Substantia Nigra and Striatum of the 6-Hydroxydopamine-Treated Rats. STEM CELLS Translational Med. 8 (5), 490-499. doi:10.1002/sctm.18-0162

Phinney, D. G., and Pittenger, M. F. (2017). Concise Review: MSC-Derived Exosomes for Cell-free Therapy. Stem Cells 35 (4), 851-858. doi:10.1002/ stem. 2575

Pittenger, M. F., Discher, D. E., Péault, B. M., Phinney, D. G., Hare, J. M., and Caplan, A. I. (2019). Mesenchymal Stem Cell Perspective: Cell Biology to Clinical Progress. NPJ Regen. Med. 4, 22. doi:10.1038/s41536-019-0083-6

Pivoraitè, U., Jarmalavičiūte, A., Tunaitis, V., Ramanauskaitè, G., Vaitkuvienè, A., Kašèta, V., et al. (2015). Exosomes from Human Dental Pulp Stem Cells Suppress Carrageenan-Induced Acute Inflammation in Mice. Inflammation 38 (5), 1933-1941. doi:10.1007/s10753-015-0173-6

Rao, F., Zhang, D., Fang, T., Lu, C., Wang, B., Ding, X., et al. (2019). Exosomes from Human Gingiva-Derived Mesenchymal Stem Cells Combined with Biodegradable Chitin Conduits Promote Rat Sciatic Nerve Regeneration. Stem Cell Int. 2019, 1-12. doi:10.1155/2019/2546367

Rosa, V., Zhang, Z., Grande, R. H. M., and Nör, J. E. (2013). Dental Pulp Tissue Engineering in Full-Length Human Root Canals. J. Dent Res. 92 (11), 970-975. doi: 10.1177/0022034513505772

Ryu, K.-H., Cho, K.-A., Park, H. S., Kim, J.-Y., Woo, S.-Y., Jo, I., et al. (2012). Tonsil-derived Mesenchymal Stromal Cells: Evaluation of Biologic, Immunologic and Genetic Factors for Successful Banking. Cytotherapy 14 (10), 1193-1202. doi:10.3109/14653249.2012.706708

Secco, M., Zucconi, E., Vieira, N. M., Fogaça, L. L. Q., Cerqueira, A., Carvalho, M. D. F., et al. (2008). Multipotent Stem Cells from Umbilical Cord: Cord Is Richer Than Blood!. Stem Cells 26 (1), 146-150. doi:10.1634/ stemcells.2007-0381

Sedgley, C. M., and Botero, T. M. (2012). Dental Stem Cells and Their Sources. Dental Clin. North America 56 (3), 549-561. doi:10.1016/j.cden.2012.05.004

Shen, Z., Kuang, S., Zhang, Y., Yang, M., Qin, W., Shi, X., et al. (2020). Chitosan Hydrogel Incorporated with Dental Pulp Stem Cell-Derived Exosomes Alleviates Periodontitis in Mice via a Macrophage-dependent Mechanism. Bioactive Mater. 5 (4), 1113-1126. doi:10.1016/j.bioactmat.2020.07.002

Shi, Q., Qian, Z., Liu, D., Sun, J., Wang, X., Liu, H., et al. (2017). GMSC-derived Exosomes Combined with a Chitosan/Silk Hydrogel Sponge Accelerates Wound Healing in a Diabetic Rat Skin Defect Model. Front. Physiol. 8, 904. doi:10.3389/fphys.2017.00904

Sonoyama, W., Liu, Y., Fang, D., Yamaza, T., Seo, B.-M., Zhang, C., et al. (2006). Mesenchymal Stem Cell-Mediated Functional Tooth Regeneration in Swine. PLoS One 1, e79. doi:10.1371/journal.pone.0000079

Sonoyama, W., Liu, Y., Yamaza, T., Tuan, R. S., Wang, S., Shi, S., et al. (2008). Characterization of the Apical Papilla and its Residing Stem Cells from Human Immature Permanent Teeth: a Pilot Study. J. Endodontics 34 (2), 166-171. doi:10.1016/j.joen.2007.11.021

Stanko, P., Altanerova, U., Jakubechova, J., Repiska, V., and Altaner, C. (2018). Dental Mesenchymal Stem/Stromal Cells and Their Exosomes. Stem Cell Int. 2018, 1-8. doi:10.1155/2018/8973613

Swanson, W. B., Gong, T., Zhang, Z., Eberle, M., Niemann, D., Dong, R., et al. (2020). Controlled Release of Odontogenic Exosomes from a Biodegradable Vehicle Mediates Dentinogenesis as a Novel Biomimetic Pulp Capping Therapy. J. Controlled Release 324, 679-694. doi:10.1016/ j.jconrel.2020.06.006

Tomokiyo, A., Wada, N., and Maeda, H. (2019). Periodontal Ligament Stem Cells: Regenerative Potency in Periodontium. Stem Cell Develop. 28 (15), 974-985. doi:10.1089/scd.2019.0031

Tsubokawa, T., Yagi, K., Nakanishi, C., Zuka, M., Nohara, A., Ino, H., et al. (2010). Impact of Anti-apoptotic and Anti-oxidative Effects of Bone Marrow Mesenchymal Stem Cells with Transient Overexpression of Heme Oxygenase-1 on Myocardial Ischemia. Am. J. Physiology-Heart Circulatory Physiol. 298 (5), H1320-H1329. doi:10.1152/ajpheart.01330.2008

Uccelli, A., Moretta, L., and Pistoia, V. (2008). Mesenchymal Stem Cells in Health and Disease. Nat. Rev. Immunol. 8 (9), 726-736. doi:10.1038/nri2395

Vakhshiteh, F., Rahmani, S., Ostad, S. N., Madjd, Z., Dinarvand, R., and Atyabi, F. (2021). Exosomes Derived from miR-34a-Overexpressing Mesenchymal Stem
Cells Inhibit In Vitro Tumor Growth: A New Approach for Drug Delivery. Life Sci. 266, 118871. doi:10.1016/j.lfs.2020.118871

Venugopal, C., Shobha, K., Rai, K. S., Pinnelli, V. B., Kutty, B. M., and Dhanushkodi, A. (2018). Neuroprotection by Human Dental Pulp Mesenchymal Stem Cells: From Billions to Nano. Cgt 18 (5), 307-323. doi:10.2174/1566523218666180913152615

Victor, A. K., and Reiter, L. T. (2017). Dental Pulp Stem Cells for the Study of Neurogenetic Disorders. Hum. Mol. Genet. 26 (R2), R166-R171. doi:10.1093/ $\mathrm{hmg} / \mathrm{ddx} 208$

Vlassov, A. V., Magdaleno, S., Setterquist, R., and Conrad, R. (2012). Exosomes: Current Knowledge of Their Composition, Biological Functions, and Diagnostic and Therapeutic Potentials. Biochim. Biophys. Acta (Bba) - Gen. Subjects 1820 (7), 940-948. doi:10.1016/j.bbagen.2012.03.017

Wang, A., Liu, J., Zhuang, X., Yu, S., Zhu, S., Liu, Y., et al. (2020a). Identification and Comparison of piRNA Expression Profiles of Exosomes Derived from Human Stem Cells from the Apical Papilla and Bone Marrow Mesenchymal Stem Cells. Stem Cell Develop. 29 (8), 511-520. doi:10.1089/scd.2019.0277

Wang, D., Wang, Y., Tian, W., and Pan, J. (2019). Advances of Tooth-derived Stem Cells in Neural Diseases Treatments and Nerve Tissue Regeneration. Cell Prolif 52 (3), e12572. doi:10.1111/cpr.12572

Wang, M., Li, J., Ye, Y., He, S., and Song, J. (2020b). SHED-derived Conditioned Exosomes Enhance the Osteogenic Differentiation of PDLSCs via Wnt and BMP Signaling In Vitro. Differentiation 111, 1-11. doi:10.1016/ j.diff.2019.10.003

Wei, J., Song, Y., Du, Z., Yu, F., Zhang, Y., Jiang, N., et al. (2020). Exosomes Derived from Human Exfoliated Deciduous Teeth Ameliorate Adult Bone Loss in Mice through Promoting Osteogenesis. J. Mol. Hist. 51 (4), 455-466. doi:10.1007/ s10735-020-09896-3

Wolf, P. (1967). The Nature and Significance of Platelet Products in Human Plasma. Br. J. Haematol. 13 (3), 269-288. doi:10.1111/j.13652141.1967.tb08741.x

Wu, J., Chen, L., Wang, R., Song, Z., Shen, Z., Zhao, Y., et al. (2019). Exosomes Secreted by Stem Cells from Human Exfoliated Deciduous Teeth Promote Alveolar Bone Defect Repair through the Regulation of Angiogenesis and Osteogenesis. ACS Biomater. Sci. Eng. 5 (7), 3561-3571. doi:10.1021/ acsbiomaterials.9b00607

Xie, L., Chen, J., Ren, X., Zhang, M., Thuaksuban, N., Nuntanaranont, T., et al. (2021). Alteration of circRNA and lncRNA Expression Profile in Exosomes Derived from Periodontal Ligament Stem Cells Undergoing Osteogenic Differentiation. Arch. Oral Biol. 121, 104984. doi:10.1016/ j.archoralbio.2020.104984

Xu, F., Zhong, J. Y., Lin, X., Shan, S. K., Guo, B., Zheng, M. H., et al. (2020a). Melatonin Alleviates Vascular Calcification and Ageing through Exosomal miR-204/miR-211 Cluster in a Paracrine Manner. J. Pineal Res. 68 (3), e12631. doi:10.1111/jpi.12631

Xu, X. Y., Tian, B. M., Xia, Y., Xia, Y. L., Li, X., Zhou, H., et al. (2020b). Exosomes Derived from P2X7 Receptor Gene-modified Cells rescue Inflammationcompromised Periodontal Ligament Stem Cells from Dysfunction. Stem Cell Transl Med 9 (11), 1414-1430. doi:10.1002/sctm.19-0418

Yoon, Y. M., Lee, J. H., Song, K. H., Noh, H., and Lee, S. H. (2020). Melatoninstimulated Exosomes Enhance the Regenerative Potential of Chronic Kidney Disease-derived Mesenchymal Stem/stromal Cells via Cellular Prion Proteins. J. Pineal Res. 68 (3), e12632. doi:10.1111/jpi.12632

Zhang, Q., Shi, S., Liu, Y., Uyanne, J., Shi, Y., Shi, S., et al. (2009). Mesenchymal Stem Cells Derived from Human Gingiva Are Capable of Immunomodulatory Functions and Ameliorate Inflammation-Related Tissue Destruction in Experimental Colitis. J. Immunol. 183 (12), 7787-7798. doi:10.4049/ jimmunol.0902318

Zhang, Y., Bi, J., Huang, J., Tang, Y., Du, S., and Li, P. (2020a). Exosome: A Review of its Classification, Isolation Techniques, Storage, Diagnostic and Targeted Therapy Applications. Ijn 15, 6917-6934. doi:10.2147/IJN.S264498

Zhang, Y., Shi, S., Xu, Q., Zhang, Q., Shanti, R. M., and Le, A. D. (2019). SIS-ECM Laden with GMSC-Derived Exosomes Promote Taste Bud Regeneration. J. Dent Res. 98 (2), 225-233. doi:10.1177/0022034518804531

Zhang, Y., Wang, Z., Shi, B., Li, Y., Wang, R., Sun, J., et al. (2021a). Effect of Gingival Mesenchymal Stem Cell-Derived Exosomes on Inflammatory Macrophages in a High-Lipid Microenvironment. Int. Immunopharmacology 94, 107455. doi:10.1016/j.intimp.2021.107455 
Zhang, Z., Deng, M., Hao, M., and Tang, J. (2021b). Periodontal Ligament Stem Cells in the Periodontitis Niche: Inseparable Interactions and Mechanisms. J. Leukoc. Biol. 110, 565-576. doi:10.1002/JLB.4MR0421-750R

Zhang, Z., Shuai, Y., Zhou, F., Yin, J., Hu, J., Guo, S., et al. (2020b). PDLSCs Regulate Angiogenesis of Periodontal Ligaments via VEGF Transferred by Exosomes in Periodontitis. Int. J. Med. Sci. 17 (5), 558-567. doi:10.7150/ijms.40918

Zheng, Y., Dong, C., Yang, J., Jin, Y., Zheng, W., Zhou, Q., et al. (2019). Exosomal microRNA-155-5p from PDLSCs Regulated Th17/Treg Balance by Targeting Sirtuin-1 in Chronic Periodontitis. J. Cel Physiol 234 (11), 20662-20674. doi:10.1002/jcp.28671

Zhu, Y., Zhang, P., Gu, R. L., Liu, Y. S., and Zhou, Y. S. (2018). Origin and Clinical Applications of Neural Crest-Derived Dental Stem Cells. Chin. J. Dent Res. 21 (2), 89-100. doi:10.3290/j.cjdr.a40435

Zhuang, X., Ji, L., Jiang, H., Liu, Y., Liu, X., Bi, J., et al. (2020). Exosomes Derived from Stem Cells from the Apical Papilla Promote Dentine-Pulp Complex Regeneration by Inducing Specific Dentinogenesis. Stem Cell Int. 2020, 1-10. doi: $10.1155 / 2020 / 5816723$
Conflict of Interest: The authors declare that the research was conducted in the absence of any commercial or financial relationships that could be construed as a potential conflict of interest.

Publisher's Note: All claims expressed in this article are solely those of the authors and do not necessarily represent those of their affiliated organizations, or those of the publisher, the editors and the reviewers. Any product that may be evaluated in this article, or claim that may be made by its manufacturer, is not guaranteed or endorsed by the publisher.

Copyright (c) $2021 \mathrm{Mai}$, Chen, Ye, Hu, Sun, Cui and Zhao. This is an open-access article distributed under the terms of the Creative Commons Attribution License (CC $B Y)$. The use, distribution or reproduction in other forums is permitted, provided the original author(s) and the copyright owner(s) are credited and that the original publication in this journal is cited, in accordance with accepted academic practice. No use, distribution or reproduction is permitted which does not comply with these terms. 